\title{
ESTIMATE FOR INITIAL MACLAURIN COEFFICIENTS OF CERTAIN SUBCLASSES OF BI-UNIVALENT FUNCTIONS
}

\author{
BADR S. ALKAHTANI, PRANAY GOSWAMI, AND TEODOR BULBOACĂ
}

Received 26 February, 2015

\begin{abstract}
In this paper, estimates for second and third MacLaurin coefficients of certain subclasses of bi-univalent functions in the open unit disk defined by convolution are determined, and certain special cases are also indicated. The main result extends and improve a recent one obtained by Srivastava et al.
\end{abstract}

2010 Mathematics Subject Classification: 30C45; 30C50

Keywords: univalent functions, bi-univalent functions, bi-starlike function, bi-convex function, functions with bounded boundary rotation, coefficient estimates, convolution (Hadamard) product

\section{INTRODUCTION AND DEFINITIONS}

Let $\mathcal{A}$ be the class of functions $f$ of the form $f(z)=z+\sum_{n=2}^{\infty} a_{n} z^{n}$, which are analytic in the open unit disk $\mathbb{D}=\{z \in \mathbb{C}:|z|<1\}$ and normalized by the conditions $f(0)=0$ and $f^{\prime}(0)=1$. The Koebe one-quarter theorem [3] ensures that the image of $\mathbb{D}$ under every univalent function $f \in \mathcal{A}$ contains the disk with the center in the origin and the radius $1 / 4$. Thus, every univalent function $f \in \mathcal{A}$ has an inverse $f^{-1}: f(\mathbb{D}) \rightarrow \mathbb{D}$, satisfying $f^{-1}(f(z))=z, z \in \mathbb{D}$, and

$$
f\left(f^{-1}(w)\right)=w,|w|<r_{0}(f), r_{0}(f) \geq \frac{1}{4} .
$$

Moreover, it is easy to see that the inverse function has the series expansion of the form

$$
f^{-1}(w)=w-a_{2} w^{2}+\left(2 a_{2}^{2}-a_{3}\right) w^{3}-\left(5 a_{2}^{3}-5 a_{2} a_{3}+a_{4}\right) w^{4}+\ldots, w \in f(\mathbb{D}) .
$$

A function $f \in \mathcal{A}$ is said to be bi-univalent, if both $f$ and $f^{-1}$ are univalent in $\mathbb{D}$, in the sense that $f^{-1}$ has a univalent analytic continuation to $\mathbb{D}$, and we denote by $\sigma$ this class of bi-univalent functions. 
In [8] the authors defined the classes of functions $\mathcal{P}_{m}(\beta)$ as follows: let $\mathcal{P}_{m}(\beta)$, with $m \geq 2$ and $0 \leq \beta<1$, denote the class of univalent analytic functions $P$, normalized with $P(0)=1$, and satisfying

$$
\int_{0}^{2 \pi}\left|\frac{\operatorname{Re} P(z)-\beta}{1-\beta}\right| \mathrm{d} \theta \leq m \pi,
$$

where $z=r e^{i \theta} \in \mathbb{D}$.

For $\beta=0$, we denote $\mathcal{P}_{m}:=\mathcal{P}_{m}(0)$, hence the class $\mathcal{P}_{m}$ represents the class of functions $p$ analytic in $\mathbb{D}$, normalized with $p(0)=1$, and having the representation

$$
p(z)=\int_{0}^{2 \pi} \frac{1-z e^{i t}}{1+z e^{i t}} \mathrm{~d} \mu(t),
$$

where $\mu$ is a real-valued function with bounded variation, which satisfies

$$
\int_{0}^{2 \pi} d \mu(t)=2 \pi \quad \text { and } \quad \int_{0}^{2 \pi}|d \mu(t)| \leq m, m \geq 2 .
$$

Clearly, $\mathcal{P}:=\mathcal{P}_{2}$ is the well-known class of Carathéodory functions, i.e. the normalized functions with positive real part in the open unit disk $\mathbb{D}$.

Lewin [6] investigated the class $\sigma$ of bi-univalent functions and obtained the bound for the second coefficient. Brannan and Taha [2] considered certain subclasses of biunivalent functions, similar to the familiar subclasses of univalent functions consisting of strongly starlike, starlike and convex functions. They introduced the concept of bi-starlike functions and the bi-convex functions, and obtained estimates for the initial coefficients. Recently, Ali et al. [1], Srivastava et al. [9], Frasin and Aouf [4], Goyal and Goswami [5] and many others have introduced and investigated subclasses of bi-univalent functions and obtained bounds for the initial coefficients. Motivated by work of Srivastava et al. [9], we introduce a new subclass of bi-univalent functions, as follows.

For the functions $f, h \in \mathcal{A}$ given by

$$
f(z)=z+\sum_{n=2}^{\infty} a_{n} z^{n}, \quad h(z)=z+\sum_{n=2}^{\infty} b_{n} z^{n}, z \in \mathbb{D},
$$

we recall the Hadamard (or convolution) product of $f$ and $h$, defined by

$$
(f * h)(z)=z+\sum_{n=2}^{\infty} a_{n} b_{n} z^{n}, z \in \mathbb{D} .
$$

Definition 1. For a given function $k \in \sigma$, a function $f \in \sigma$ is said to be in the class $\mathscr{B} \mathcal{R}^{k}(m ; \beta)$, with $m \geq 2$ and $0 \leq \beta<1$, if the following conditions are satisfied

$$
\frac{(f * k)(z)}{z} \in \mathcal{P}_{m}(\beta)
$$




$$
\frac{(g * k)(w)}{w} \in \mathcal{P}_{m}(\beta)
$$

where $g=f^{-1}$ and $z, w \in \mathbb{D}$.

Remark 1. Taking $k(z)=z /(1-z)^{2}$ and $m=2$ in the Definition 1 we obtain the class $\mathcal{B}(\beta):=\mathscr{B}^{z /(1-z)^{2}}(2 ; \beta)$ studied by Srivastava et al. [9, Definition 2].

Definition 2. For a given function $k \in \sigma$ and a number $\alpha \in \mathbb{C}$, a function $f \in \sigma$ is said to be in the class $\mathcal{B} \mathcal{V}^{k}(m ; \alpha, \beta)$, with $m \geq 2$ and $0 \leq \beta<1$, if the following conditions are satisfied

$$
\begin{aligned}
& (1-\alpha) \frac{z(f * k)^{\prime}(z)}{(f * k)(z)}+\alpha\left(1+\frac{z(f * k)^{\prime \prime}(z)}{(f * k)^{\prime}(z)}\right) \in \mathcal{P}_{m}(\beta), \\
& (1-\alpha) \frac{w(g * k)^{\prime}(w)}{(g * k)(w)}+\alpha\left(1+\frac{w(g * k)^{\prime \prime}(w)}{(g * k)^{\prime}(w)}\right) \in \mathcal{P}_{m}(\beta),
\end{aligned}
$$

where $g=f^{-1}$.

Remark 2. (i) Taking $\alpha=0$ and $\alpha=1$ in the above class $B \mathcal{V}^{k}(m ; \alpha, \beta)$ we obtain the classes $\delta_{m}^{k}(\beta):=\mathscr{B} \mathcal{V}^{k}(m ; 0, \beta)$ and $\ell_{m}^{k}(\beta):=\mathscr{B} \mathcal{V}^{k}(m ; 1, \beta)$, respectively.

(i i ) Moreover, if we take $k(z)=z /(1-z)$ and $m=2$, the classes $g_{m}^{k}(\beta)$ and $\varphi_{m}^{k}(\beta)$ reduces to the well-known classes of bi-starlike and bi-convex functions, respectively (see also [2]).

The object of the paper is to find estimates for the coefficients $a_{2}$ and $a_{3}$ for functions in the subclass $\mathcal{B} \mathcal{R}^{k}(m ; \beta)$ and $\mathcal{B} \mathcal{V}^{k}(m ; \alpha, \beta)$, and these bounds are obtained by employing the techniques used earlier by Srivastava et al. [9].

\section{MAIN RESULTS}

In order to prove our main result for the functions $f \in \mathscr{B} \mathcal{R}^{k}(m ; \beta)$, first we will prove the following lemma:

Lemma 1. Let the function $\Phi(z)=1+\sum_{n=1}^{\infty} h_{n} z^{n}, z \in \mathbb{D}$, such that $\Phi \in \mathcal{P}_{m}(\beta)$. Then,

$$
\left|h_{n}\right| \leq m(1-\beta), n \geq 1 .
$$

Proof. From (1.2) and (1.3), like in [8] and [7], we can see that if $p \in \mathcal{P}_{m}$, then

$$
p(z)=\left(\frac{m}{4}+\frac{1}{2}\right) p_{1}(z)-\left(\frac{m}{4}-\frac{1}{2}\right) p_{2}(z),
$$

where $p_{1}, p_{2} \in \mathcal{P}$. 
Further, if $p(z)=1+\sum_{n=1}^{\infty} p_{n} z^{n}, z \in \mathbb{D}$, where $p_{1}(z)=1+\sum_{n=1}^{\infty} p_{n}^{(1)} z^{n}$ and $p_{2}(z)=1+\sum_{n=1}^{\infty} p_{n}^{(2)} z^{n}$ for all $z \in \mathbb{D}$, comparing the coefficients of both sides of (2.1) we get

$$
p_{n}=\left(\frac{m}{4}+\frac{1}{2}\right) p_{n}^{(1)}-\left(\frac{m}{4}-\frac{1}{2}\right) p_{n}^{(2)}, n \geq 1 .
$$

Since $p_{1}, p_{2} \in \mathcal{P}$, where $\mathcal{P}$ is the class of Carathéodory functions, it is well-known that $\left|p_{n}^{(1)}\right| \leq 2$ and $\left|p_{n}^{(2)}\right| \leq 2$ for all $n \geq 1$, and thus

$$
\begin{gathered}
\left|p_{n}\right| \leq\left(\frac{m}{4}+\frac{1}{2}\right)\left|p_{n}^{(1)}\right|+\left(\frac{m}{4}-\frac{1}{2}\right)\left|p_{n}^{(2)}\right| \leq \\
2\left(\frac{m}{4}+\frac{1}{2}\right)+2\left(\frac{m}{4}-\frac{1}{2}\right)=m, n \geq 1 .
\end{gathered}
$$

Now, the proof of this lemma is straight forward, if we write

$$
\Phi(z)=(1-\beta) p(z)+\beta, \quad \text { where } \quad p(z)=1+\sum_{n=1}^{\infty} p_{n} z^{n} \in \mathcal{P}_{m} .
$$

Then,

$$
\Phi(z)=1+(1-\beta) \sum_{n=1}^{\infty} p_{n} z^{n}, z \in \mathbb{D}
$$

which gives

$$
h_{n}=(1-\beta) p_{n}, n \geq 1,
$$

and using the inequality (2.2) we obtain the desired result.

Theorem 1. Let $f(z)=z+\sum_{n=2}^{\infty} a_{n} z^{n}$ be in the class $\mathcal{B}^{k}(m ; \beta)$, where $k \in \sigma$ has the form $k(z)=z+\sum_{n=2}^{\infty} k_{n} z^{n}$. If $k_{2}, k_{3} \neq 0$, then

$$
\left|a_{2}\right| \leq \min \left\{\sqrt{\frac{m(1-\beta)}{\left|k_{3}\right|}} ; \frac{m(1-\beta)}{\left|k_{2}\right|}\right\}, \quad\left|a_{3}\right| \leq \frac{m(1-\beta)}{\left|k_{3}\right|},
$$

and

$$
\left|2 a_{2}^{2}-a_{3}\right| \leq \frac{m(1-\beta)}{\left|k_{3}\right|} .
$$

Proof. Since $f \in \mathscr{B} \mathcal{R}^{k}(m ; \beta)$, from the Definition 1 we have

$$
\frac{(f * k)(z)}{z}=p(z)
$$


and

$$
\frac{(g * k)(w)}{w}=q(w),
$$

where $p, q \in \mathcal{P}_{m}(\beta)$ and $g=f^{-1}$. Using the fact that the functions $p$ and $q$ have the following Taylor expansions

$$
\begin{aligned}
& p(z)=1+p_{1} z+p_{2} z^{2}+p_{3} z^{3}+\ldots, z \in \mathbb{D}, \\
& q(w)=1+q_{1} w+q_{2} w^{2}+q_{3} w^{3}+\ldots, w \in \mathbb{D},
\end{aligned}
$$

and equating the coefficients in (2.3) and (2.4), from (1.1) we get

$$
\begin{aligned}
& k_{2} a_{2}=p_{1}, \\
& k_{3} a_{3}=p_{2},
\end{aligned}
$$

and

$$
\begin{aligned}
& -k_{2} a_{2}=q_{1}, \\
& k_{3}\left(2 a_{2}^{2}-a_{3}\right)=q_{2} .
\end{aligned}
$$

Since $p, q \in \mathcal{P}_{m}(\beta)$, according to Lemma 1 , the next inequalities hold:

$$
\begin{aligned}
& \left|p_{k}\right| \leq m(1-\beta), k \geq 1 \\
& \left|q_{k}\right| \leq m(1-\beta), k \geq 1
\end{aligned}
$$

and thus, from (2.8) and (2.9), by using the inequalities (2.10) and (2.11), we obtain

which gives

$$
\left|a_{2}\right|^{2} \leq \frac{\left|q_{2}\right|+\left|p_{2}\right|}{2\left|k_{3}\right|} \leq \frac{m(1-\beta)}{\left|k_{3}\right|}
$$

$$
\left|a_{2}\right| \leq \sqrt{\frac{m(1-\beta)}{\left|k_{3}\right|}} .
$$

From (2.7), by using (2.10) we obtain immediately that

$$
\left|a_{2}\right|=\left|\frac{p_{1}}{k_{2}}\right| \leq \frac{m(1-\beta)}{\left|k_{2}\right|},
$$

and combining this with the inequality (2.12), the first inequality of the conclusion is proved.

According to (2.8), from (2.10) we easily obtain

$$
\left|a_{3}\right|=\left|\frac{p_{2}}{k_{3}}\right| \leq \frac{m(1-\beta)}{\left|k_{3}\right|},
$$

and from (2.9), by using (2.10) and (2.11) we finally deduce

$$
\left|2 a_{2}^{2}-a_{3}\right|=\left|\frac{q_{2}}{k_{3}}\right| \leq \frac{m(1-\beta)}{\left|k_{3}\right|},
$$

which completes our proof. 
Setting $\beta=0$ in Theorem 1 we get the following special case:

Corollary 1. Let $f(z)=z+\sum_{n=2}^{\infty} a_{n} z^{n}$ be in the class $\mathcal{B}^{k}(m ; 0)$, where $k \in \sigma$ has the form $k(z)=z+\sum_{n=2}^{\infty} k_{n} z^{n}$. If $k_{2}, k_{3} \neq 0$, then

$$
\left|a_{2}\right| \leq \min \left\{\sqrt{\frac{m}{\left|k_{3}\right|}} ; \frac{m}{\left|k_{2}\right|}\right\}, \quad\left|a_{3}\right| \leq \frac{m}{\left|k_{3}\right|}, \quad \text { and } \quad\left|2 a_{2}^{2}-a_{3}\right| \leq \frac{m}{\left|k_{3}\right|} .
$$

For $k(z)=z /(1-z)^{2}$ the above corollary reduces to the next result:

Example 1. If $f(z)=z+\sum_{n=2}^{\infty} a_{n} z^{n}$ is in the class $\mathcal{B} \mathcal{R}^{z /(1-z)^{2}}(m ; 0)$, then

$$
\left|a_{2}\right| \leq \sqrt{\frac{m}{3}}, \quad\left|a_{3}\right| \leq \frac{m}{3}, \quad \text { and } \quad\left|2 a_{2}^{2}-a_{3}\right| \leq \frac{m}{3} .
$$

Taking $k(z)=z /(1-z)$ in Corollary 1 , we get:

Example 2. If $f(z)=z+\sum_{n=2}^{\infty} a_{n} z^{n}$ is in the class $\mathcal{B} \mathcal{R}^{z /(1-z)}(m ; 0)$, then

$$
\left|a_{2}\right| \leq \sqrt{m}, \quad\left|a_{3}\right| \leq m, \quad \text { and } \quad\left|2 a_{2}^{2}-a_{3}\right| \leq m .
$$

If we put $k(z)=z /(1-z)^{2}$ in Theorem 1 , we deduce the next corollary:

Corollary 2. If $f(z)=z+\sum_{n=2}^{\infty} a_{n} z^{n}$ is in the class $B(\beta)$, then

$$
\left|a_{2}\right| \leq\left\{\begin{array}{ll}
\sqrt{\frac{2(1-\beta)}{3}}, & \text { if } \quad 0 \leq \beta \leq \frac{1}{3}, \\
1-\beta, & \text { if } \quad \frac{1}{3}<\beta<1,
\end{array} \quad\left|a_{3}\right| \leq \frac{2(1-\beta)}{3},\right.
$$

and

$$
\left|2 a_{2}^{2}-a_{3}\right| \leq \frac{2(1-\beta)}{3} .
$$

Remark 3. For the special case $\frac{1}{3}<\beta<1$, the above first inequality, and the second one for all $0 \leq \beta<1$, improve the estimates given by Srivastava et al. in [9, Theorem 2].

Theorem 2. Let $f(z)=z+\sum_{n=2}^{\infty} a_{n} z^{n}$ be in the class $\mathcal{B V}^{k}(m ; \alpha, \beta)$, with $\alpha \in$ $\mathbb{C} \backslash\{-1\}$, where $k \in \sigma$ has the form $k(z)=z+\sum_{n=2}^{\infty} k_{n} z^{n}$. If $k_{2}, k_{3} \neq 0$ and

$$
2(1+2 \alpha) k_{3}-(1+3 \alpha) k_{2}^{2} \neq 0 \text {, }
$$


then

$$
\left|a_{2}\right| \leq \min \left\{\sqrt{\frac{m(1-\beta)}{\left|2(1+2 \alpha) k_{3}-(1+3 \alpha) k_{2}^{2}\right|}} ; \frac{m(1-\beta)}{|1+\alpha|\left|k_{2}\right|}\right\},
$$

and

$$
\begin{array}{r}
\left|a_{3}\right| \leq \min \left\{\frac{m(1-\beta)}{\left|2(1+2 \alpha) k_{3}-(1+3 \alpha) k_{2}^{2}\right|}+\frac{m(1-\beta)}{2|1+2 \alpha|\left|k_{3}\right|} ;\right. \\
\frac{m(1-\beta)}{2|1+2 \alpha|\left|k_{3}\right|}\left(1+\frac{m(1-\beta)|1+3 \alpha|}{|1+\alpha|^{2}}\right) ; \\
\left.\frac{m(1-\beta)}{2|1+2 \alpha|\left|k_{3}\right|}\left(1+\frac{m(1-\beta)\left|4(1+2 \alpha) k_{3}-(1+3 \alpha) k_{2}^{2}\right|}{\left|k_{2}\right|^{2}|1+\alpha|^{2}}\right)\right\},
\end{array}
$$

whenever $\alpha \in \mathbb{C} \backslash\left\{-\frac{1}{2}\right\}$.

Proof. If $f \in B \mathcal{V}^{k}(m ; \alpha, \beta)$, according to the Definition 2 we have

$$
(1-\alpha) \frac{z(f * k)^{\prime}(z)}{(f * k)(z)}+\alpha\left(1+\frac{z(f * k)^{\prime \prime}(z)}{(f * k)^{\prime}(z)}\right)=p(z)
$$

and

$$
(1-\alpha) \frac{w(g * k)^{\prime}(w)}{(g * k)(w)}+\alpha\left(1+\frac{w(g * k)^{\prime \prime}(w)}{(g * k)^{\prime}(w)}\right)=q(w),
$$

where $p, q \in \mathcal{P}_{m}(\beta)$ and $g=f^{-1}$. Since

$$
\begin{aligned}
& (1-\alpha) \frac{z(f * k)^{\prime}(z)}{(f * k)(z)}+\alpha\left(1+\frac{z(f * k)^{\prime \prime}(z)}{(f * k)^{\prime}(z)}\right)= \\
& 1+(1+\alpha) a_{2} k_{2} z+\left[2(1+2 \alpha) a_{3} k_{3}-(1+3 \alpha) a_{2}^{2} k_{2}^{2}\right] z^{2}+\ldots z \in \mathbb{D},
\end{aligned}
$$

and according to $(1.1)$

$$
\begin{aligned}
& (1-\alpha) \frac{z(g * k)^{\prime}(w)}{(g * k)(w)}+\alpha\left(1+\frac{z(g * k)^{\prime \prime}(w)}{(g * k)^{\prime}(w)}\right)=1-(1+\alpha) a_{2} k_{2} w+ \\
& \left\{\left[4(1+2 \alpha) k_{3}-(1+3 \alpha) k_{2}^{2}\right] a_{2}^{2}-2(1+2 \alpha) a_{3} k_{3}\right\} w^{2}+\ldots, w \in \mathbb{D},
\end{aligned}
$$

from (2.5) and (2.6) combined with the above two expansion formulas, it follows that

$$
\begin{aligned}
& (1+\alpha) a_{2} k_{2}=p_{1}, \\
& 2(1+2 \alpha) a_{3} k_{3}-(1+3 \alpha) a_{2}^{2} k_{2}^{2}=p_{2},
\end{aligned}
$$

and

$$
\begin{aligned}
& -(1+\alpha) a_{2} k_{2}=q_{1}, \\
& {\left[4(1+2 \alpha) k_{3}-(1+3 \alpha) k_{2}^{2}\right] a_{2}^{2}-2(1+2 \alpha) a_{3} k_{3}=q_{2} .}
\end{aligned}
$$


Now, from (2.14) and (2.15) we deduce that

$$
a_{2}^{2}=\frac{p_{2}+q_{2}}{4(1+2 \alpha) k_{3}-2(1+3 \alpha) k_{2}^{2}}
$$

whenever $2(1+2 \alpha) k_{3}-(1+3 \alpha) k_{2}^{2} \neq 0$, and

$$
4(1+2 \alpha) k_{3}\left(a_{3}-a_{2}^{2}\right)=p_{2}-q_{2} \text {. }
$$

Using (2.16) in the above relation, we obtain

$$
\begin{aligned}
& a_{3}=\frac{p_{2}+q_{2}}{4(1+2 \alpha) k_{3}-2(1+3 \alpha) k_{2}^{2}}+\frac{p_{2}-q_{2}}{4(1+2 \alpha) k_{3}}, \\
& \text { whenever } 2(1+2 \alpha) k_{3}-(1+3 \alpha) k_{2}^{2} \neq 0, \alpha \in \mathbb{C} \backslash\left\{-\frac{1}{2}\right\} .
\end{aligned}
$$

From (2.13) and (2.14) we get

$$
a_{3}=\frac{1}{2(1+2 \alpha) k_{3}}\left[p_{2}+\frac{1+3 \alpha}{(1+\alpha)^{2}} p_{1}^{2}\right], \quad \text { for } \quad \alpha \in \mathbb{C} \backslash\left\{-1 ;-\frac{1}{2}\right\},
$$

while from (2.13) and (2.15) we deduce that

$$
a_{3}=\frac{1}{2(1+2 \alpha) k_{3}}\left[-q_{2}+\frac{4(1+2 \alpha) k_{3}-(1+3 \alpha) k_{2}^{2}}{k_{2}^{2}(1+\alpha)^{2}} p_{1}^{2}\right],
$$

for $\alpha \in \mathbb{C} \backslash\left\{-1 ;-\frac{1}{2}\right\}$.

Combining (2.13) and (2.16) for the computation of the upper-bound of $\left|a_{2}\right|$, and (2.17), (2.18) and (2.19) for the computation of $\left|a_{3}\right|$, by using Lemma 1 we easily find the estimates of our theorem.

Taking $\alpha=0$ and $\alpha=1$ in Theorem 2 we obtain the following two special cases, respectively:

Corollary 3. Let $f(z)=z+\sum_{n=2}^{\infty} a_{n} z^{n}$ be in the class $8_{m}^{k}(\beta)$, where $k \in \sigma$ has the form $k(z)=z+\sum_{n=2}^{\infty} k_{n} z^{n}$. If $k_{2}, k_{3} \neq 0$ and

$$
2 k_{3}-k_{2}^{2} \neq 0
$$

then

and

$$
\left|a_{2}\right| \leq \min \left\{\sqrt{\frac{m(1-\beta)}{\left|2 k_{3}-k_{2}^{2}\right|}} ; \frac{m(1-\beta)}{\left|k_{2}\right|}\right\}
$$

$$
\left|a_{3}\right| \leq \min \left\{\frac{m(1-\beta)}{\left|2 k_{3}-k_{2}^{2}\right|}+\frac{m(1-\beta)}{2\left|k_{3}\right|} ; \frac{m(1-\beta)(1+m(1-\beta))}{2\left|k_{3}\right|}\right.
$$




$$
\left.\frac{m(1-\beta)}{2\left|k_{3}\right|}\left(1+\frac{m(1-\beta)\left|4 k_{3}-k_{2}^{2}\right|}{\left|k_{2}\right|^{2}}\right)\right\} .
$$

Corollary 4. Let $f(z)=z+\sum_{n=2}^{\infty} a_{n} z^{n}$ be in the class $\varphi_{m}^{k}(\beta)$, where $k \in \sigma$ has the form $k(z)=z+\sum_{n=2}^{\infty} k_{n} z^{n}$. If $k_{2}, k_{3} \neq 0$ and

$$
3 k_{3}-2 k_{2}^{2} \neq 0
$$

then

and

$$
\left|a_{2}\right| \leq \min \left\{\sqrt{\frac{m(1-\beta)}{\left|6 k_{3}-4 k_{2}^{2}\right|}} ; \frac{m(1-\beta)}{2\left|k_{2}\right|}\right\},
$$

$$
\begin{array}{r}
\left|a_{3}\right| \leq \min \left\{\frac{m(1-\beta)}{\left|6 k_{3}-4 k_{2}^{2}\right|}+\frac{m(1-\beta)}{6\left|k_{3}\right|} ; \frac{m(1-\beta)(1+m(1-\beta))}{6\left|k_{3}\right|}\right. \\
\left.\frac{m(1-\beta)}{6\left|k_{3}\right|}\left(1+\frac{m(1-\beta)\left|3 k_{3}-k_{2}^{2}\right|}{\left|k_{2}\right|^{2}}\right)\right\} .
\end{array}
$$

\section{ACKNOWLEDGEMENT}

Dr. Badr S. Alkahtani and Dr. Pranay Goswami extend their sincere appropriations to the Deanship of Scientific Research at King Saud University for its funding this Profile Research Group (PRG-1437-35).

\section{REFERENCES}

[1] R. M. Ali, S. K. Lee, V. Ravichandran, and S. Supramaniam, "Coefficient estimates for bi-univalent Ma-Minda stalike and convex functions," Appl. Math. Lett., vol. 25, no. 3, pp. 344-351, 2012, doi: 10.1016/j.aml.2011.09.012.

[2] D. A. Brannan and T. S. Taha, "On some classes of bi-univalent functions," Stud. Univ. BabeşBolyai Math., vol. 31, no. 2, pp. 70-77, 1986.

[3] P. L. Duren, "Univalent functions," in Grundlehren der Mathematischen Wissenschaften Series. New York: Springer Verlag, 1983, vol. 259.

[4] B. A. Frasin and M. K. Aouf, "New subclasses of bi-univalent functions," Appl. Math. Lett., vol. 24, no. 9, pp. 1569-1573, 2011, doi: 10.1016/j.aml.2011.03.048.

[5] S. P. Goyal and P. Goswami, "Estimate for initial Maclaurin coefficients of bi-univalent functions for a class defined by fractional derivatives," J. Egyptian Math. Soc., vol. 20, no. 3, pp. 179-182, 2012, doi: 10.1016/j.joems.2012.08.020.

[6] M. Lewin, "On a coefficient problem for bi-univalent functions," Proc. Amer. Math. Soc., vol. 18, pp. 63-68, 1967, doi: 10.2307/2035225.

[7] K. I. Noor, W. Ul-Haq, M. Arif, and S. Mustafa, "On bounded boundary and bounded radius rotations," J. Inequal. Appl., vol. 2009, article ID: 813687, 12 pages, doi: 10.1155/2009/813687.

[8] K. Padmanabhan and R. Parvatham, "Properties of a class of functions with bounded boundary rotation," Ann. Polon. Math., vol. 31, pp. 311-323, 1975. 
[9] H. M. Srivastava, A. K. Mishra, and P. Gochhayat, "Certain subclasses of analytic and bi-univalent functions," Appl. Math. Lett., vol. 23, no. 10, pp. 1188-1192, 2010, doi: 10.1016/j.aml.2010.05.009.

Authors' addresses

Badr S. Alkahtani

King Saud University, Mathematics Department, College of Science, P. O. Box 1142, Riyadh 11989, Saudi Arabia

E-mail address: alhaghogegmail.com

Pranay Goswami

Ambedkar University Delhi, School of Liberal Studies, Delhi-110006, India

E-mail address: pranaygoswami83@gmail.com

Teodor Bulboacă

Babeş-Bolyai University, Faculty of Mathematics and Computer Science, 400084 Cluj-Napoca, Romania

E-mail address: bulboaca@math.ubbcluj.ro 